Article

\title{
A Generalized Index for the Assessment of Helicopter Pilot Vibration Exposure
}

\author{
Aykut Tamer $^{1}\left(\right.$, Andrea Zanoni ${ }^{2}\left(\mathbb{D},{\text { Alessandro } \operatorname{Cocco}^{2}(\mathbb{D} \text { and Pierangelo Masarati }}^{2, *} \mathbb{(}\right.$ \\ 1 Department of Mechanical Engineering, Imperial College London, London SW7 2AZ, UK; \\ a.tamer@imperial.ac.uk \\ 2 Department of Aerospace Science and Technology, Politecnico di Milano, 20156 Milano, Italy; \\ andrea.zanoni@polimi.it (A.Z.); alessandro.cocco@polimi.it (A.C.) \\ * Correspondence: pierangelo.masarati@polimi.it
}

check for

updates

Citation: Tamer, A.; Zanoni, A.;

Cocco, A.; Masarati, P. A Generalized Index for the Assessment of Helicopter Pilot Vibration Exposure. Vibration 2021, 4, 133-150. https:// doi.org/10.3390/vibration4010012

Received: 25 January 2021 Accepted: 15 February 2021 Published: 20 February 2021

Publisher's Note: MDPI stays neutral with regard to jurisdictional clai$\mathrm{ms}$ in published maps and institutional affiliations.

Copyright: (C) 2021 by the authors. Licensee MDPI, Basel, Switzerland. This article is an open access article distributed under the terms and conditions of the Creative Commons Attribution (CC BY) license (https:// creativecommons.org/licenses/by/ $4.0 /)$.

\begin{abstract}
Helicopters are known to exhibit higher vibratory levels compared to fixed-wing aircraft. The consequences of vibrations depend on the affected helicopter component or subject. Specifically, pilots are in contact with several parts of the helicopter; vibrations can spoil the pilot-vehicle interaction. To evaluate the effects of vibration exposure on pilots, comfort levels resulting from whole-body vibration are computed. However, specific body parts and organs, e.g., hands, feet, and eyes are also adversely affected, with undesirable effects on piloting quality. Therefore, a detailed assessment is necessary for a more accurate estimation of pilot vibration exposure when comparing different configurations, tracking changes during design, and determining the safety of the flight envelope. A generalized assessment is presented by considering vibrations at the seat surface, hand-grip of controls, eyes, and feet. The suggested vibration measure includes comfort, handling, feet-contact, and vision in a single formulation. It is illustrated by coupling a high-fidelity biodynamic model of the pilot to a helicopter aeroservoelastic model in a comprehensive simulation environment. Using appropriate modeling techniques, vibration exposure of helicopter pilots could be evaluated during all stages of design, to achieve a more comfortable and safer flying environment.
\end{abstract}

Keywords: human biodynamics; helicopter vibrations; helicopter comfort

\section{Introduction}

In rotorcraft, pilot- and occupant-perceived vibrations are the consequence of timedependent loads acting on the airframe. The predominant sources of vibration in helicopters are the forces and moments originating from the rotors, fuselage aerodynamics, engine. and transmission. The resulting time dependent loads excite the cockpit and the cabin and are consequently transmitted to the crew and passengers. Vibrations induce a mechanical response on the human body and may even lead to physiological and psychological reactions when of sufficiently high amplitudes [1]. Helicopter pilots are not an exception; in fact, they are more affected by the adverse effects of vibrations than cabin crew and passengers, since they are subjected to a higher workload. A first consequence is the short-term discomfort, which leads to more severe health problems in the long term, such as chronic pain [2]. Additionally, involuntary interactions as a result of vibrations on hand and control sticks can degrade handling qualities [3], which may even induce instability [4] as a result of closed loop response. Finally, visual acuity can degrade due to high frequency eye motion [5] and vibration of the instrument panels. This can increase reading errors and response times, eventually downgrading the display reading performance of the pilot.

The discussion presented above shows that vibrations may affect several body parts of a pilot. As shown in the simplified representation of Figure 1, the pilot interacts with the helicopter through the following paths: 
- $\quad$ the whole-body vibration (WBV) measured at the seat interface $\left(\ddot{z}_{s}\right)$;

- the vibration at the collective and cyclic control sticks grip $\left(\ddot{z}_{c}\right)$;

- $\quad$ the vibration of the eyes $\left(\ddot{z}_{e}\right)$;

- the involuntary motion of the feet $\left(\ddot{z}_{f}\right)$.

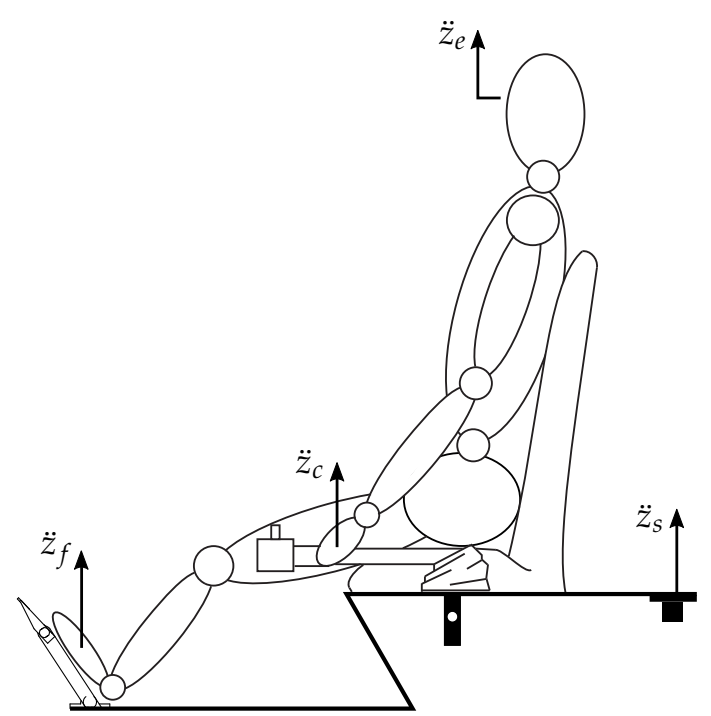

Figure 1. The four contributions of (vertical) acceleration considered in this study: seat, $\ddot{z}_{s}$; grip of collective stick, $\ddot{z}_{\mathcal{C}}$ (cyclic stick not shown); pilot's eyes, $\ddot{z}_{e}$, and feet, $\ddot{z}_{f}$.

To guide the comfort assessment, several standards have been considered: the rather general ISO-2631 [6], the aircraft focused NASA Ride Quality (RQ) [7], and the rotorcraft targeted Intrusion Index (II) from Aircraft Design Standard (ADS) 27-A [8]. ISO-2631 uses time domain accelerations at several vibration interfaces. Frequency weighting is applied in order to map the frequency-wise input level to actual, or perceived, discomfort or damage. The weighting effect can be assimilated to that of a transfer function describing how much the vibration amplitude in the specified spectrum can affect a person. In its most common and general use, ISO-2631 is applied to body vibrations measured at the seat surface, i.e., where the vibratory input enters the body. The RQ index refers to the user-defined peaks of random or sinusoidal accelerations at the cockpit floor. The II is a frequency-domain method, which is suitable for systems subjected to highly periodic inputs. Vibrations at the seat surface are taken into consideration. The use of these standards in the rotorcraft community gained pace recently. Examples include the evaluation of seat cushion designs for flight engineer seats [9], the analysis of neck strain for different flights and pilot helmet configurations [10], and evaluation of the effectiveness of vibration attenuation devices [11].

The common measures of vibration exposure generally include whole-body vibration on the seat surface, the first item in the list reported above, while the remaining three interactions are disregarded. Hence, the common comfort ratings may be insufficient to address all the affected body parts of a helicopter pilot. To address this gap, the authors suggested an index which covers vibration of whole body, eye vibration, and hand in Ref. [12]. Building on that idea, the present work proposes a generalized measure of vibration exposure in helicopter cockpit crew, which includes the contribution of the feet vibration and combines the four possible interactions listed above in a single index. Such a generalized definition, within a framework for high-fidelity simulation, is believed to be able to help tracking the effect of design changes of the helicopter on the pilots vibration exposure. By including all significant aspects that helicopter pilots face, the risk of deteriorating one contribution while improving another would be reduced. As a result, the consequences of vibrations on the occupational health of helicopter pilots and on flight safety can be thoroughly evaluated.

The document is organized as follows. Section 2 describes the contributions of the 
pilot vibration in a typical helicopter and formulates a generalized vibration index as a combination of the contributions. Section 3 describes how a detailed model is built to evaluate the suggested generalized vibration index. Section 4 demonstrates a practical application of the method and discusses its benefits. Conclusions are presented in Section 5.

\section{Method}

This section explains the steps that are required to achieve a generalized vibration index for helicopter pilots. Such index considers a combination of four subindices: (i) the comfort vibration index (CVI) that reflects the whole body response of the pilot, (ii) the handling vibration index (HVI) (iii) visual vibration index (VVI), and (iv) foot vibration index (FVI). These subindices are separately explained; subsequently, the combined index is introduced.

\subsection{Comfort Vibration Index}

The conventional approach to vibration assessment in vehicles is to measure or calculate acceleration levels at the seat surface. This reflects the response to vibrations of the body as a whole. This method of vibration assessment is also referred to as comfort rating. Among the previously mentioned alternatives, the classical ISO-2631 is found in Ref. [13] to be the most suitable in reflecting helicopter crew ratings than Intrusion Index and Ride Quality. Following the discussion therein, in the present work ISO-2631 is assumed as the conventional method for the assessment of rotorcraft crew whole-body vibration.

ISO-2631 dictates that for the assessment of whole-body vibration the measurement or computation must refer to the interface between the subject and the airframe. For most helicopter configurations and missions, this refers to the identification of translational accelerations [13] along three axes at the seat surface:

$$
\mathbf{a}_{\text {seat }}(t)=\left[\begin{array}{lll}
a_{x, \text { seat }}(t) & a_{y, \text { seat }}(t) & a_{z, \text { seat }}(t)
\end{array}\right]^{T}
$$

as a function of time $t$. However, as discussed in ISO-2631, the perception of vibration depends on frequency. Since frequency weighting cannot be applied to a temporal signal, the latter is first decomposed according to the Fourier series operation $(\mathfrak{F})$ :

$$
\left[\begin{array}{l}
A_{x}\left(\omega_{n}\right) \\
A_{y}\left(\omega_{n}\right) \\
A_{z}\left(\omega_{n}\right)
\end{array}\right]_{\text {seat }}=\frac{1}{T} \int_{-T / 2}^{+T / 2}\left[\begin{array}{l}
a_{x}(t) \\
a_{y}(t) \\
a_{z}(t)
\end{array}\right]_{\text {seat }} \mathrm{e}^{-\mathrm{j} \omega_{n} t} \mathrm{~d} t
$$

with $\omega_{n}=n \omega_{0}$, with $n \in \mathbb{Z}$, being $\omega_{0}=2 \pi / T$ the fundamental frequency of a periodic signal of period $T$.

In principle, a more general transformation, the Fourier transform, would be necessary for nonperiodic signals. However, in most practical applications, we are interested in evaluating the CVI for mission task elements (MTEs) whose duration dominates the operational time, like high-speed cruise, which, from a vibratory point of view, may be seen as substantially stationary and periodic.

The acceleration components, $A_{x, y, z}$, are now defined in the frequency domain. Then, after applying the frequency $\left(W_{d}, W_{k}\right)$ and direction $\left(k_{x}, k_{y}, k_{z}\right)$ weights defined in ISO-2631, the frequency-weighted acceleration in time domain is obtained as the sum of the frequency contributions:

$$
\mathbf{a}_{w, \text { seat }}(t)=\sum_{n=-\infty}^{n=\infty}\left[\begin{array}{l}
W_{d}\left(\omega_{n}\right) k_{x} A_{x}\left(\omega_{n}\right) \\
W_{d}\left(\omega_{n}\right) k_{y} A_{y}\left(\omega_{n}\right) \\
W_{k}\left(\omega_{n}\right) k_{z} A_{z}\left(\omega_{n}\right)
\end{array}\right] e^{j \omega_{n} t}
$$

The infinite summation in Equation (3) can be deemed impractical. However, in case 
of a dominant frequency $\omega$, the weighted acceleration becomes:

$$
a_{w, \text { seat }}(\omega)=\sqrt{\left(W_{d}(\omega) k_{x} A_{x, \omega}\right)^{2}+\left(W_{d}(\omega) k_{y} A_{y, \omega}\right)^{2}+\left(W_{k}(\omega) k_{z} A_{z, \omega}\right)^{2}}
$$

This is typical in rotorcraft; the dominant frequency would usually correspond to the angular velocity of the rotor, $\Omega$, multiplied by the number of blades, $N_{b}$, namely $\omega=N_{b} \Omega$ (Ref. [14]). It is the lowest frequency of the periodic excitation, and usually by far the largest amplitude one, that is not filtered by the rotor dynamics, and thus is entirely transmitted to the airframe. Typical values are reported in Table 1.

Table 1. Typical values of dominant frequency in helicopters, $\omega=N_{b} \Omega$.

\begin{tabular}{llcc}
\hline & $\mathbf{\Omega} \mathbf{( H z )}$ & $\boldsymbol{N}_{\boldsymbol{b}}$ & $\boldsymbol{\omega} \mathbf{( H z )}$ \\
\hline Bell B204 & 4.9 & 2 & 9.8 \\
Sikorsky H-60 Black Hawk & 4.3 & 4 & 17.2 \\
Robinson R22 & 8.8 & 2 & 17.6 \\
Mil Mi-26 & 2.2 & 8 & 17.6 \\
Aerospatiale (now Airbus) SA330 Puma & 4.5 & 4 & 18.0 \\
Sikorsky CH-53 Sea Stallion & 3.07 & 6 & 18.42 \\
Westland (now Leonardo) Lynx & 5.67 & 4 & 22.68 \\
Agusta (now Leonardo) AW139 & 4.95 & 5 & 24.75 \\
Eurocopter (now Airbus) H145 & 6.38 & 4 & 25.53 \\
Bölkow (now Airbus) BO105 & 7.07 & 4 & 28.28 \\
\hline
\end{tabular}

Equation (4) can be further simplified considering that the vertical component usually dominates in helicopters, thus yielding

$$
a_{w, \text { seat }}(\omega)=W_{k}(\omega) A_{z, \omega}
$$

where $W_{k}(\omega)$ as a function of the frequency is shown in Figure 2 and, according to ISO$2631, k_{z}=1$ for a seated person ([6], Section 7.2.3). Incidentally, it is worth noticing that, according to Figure 2, the range of frequencies of maximum sensitivity is well centered around the typical dominant frequencies of helicopter main rotors reported in Table 1.

The above weighted acceleration can be further averaged by considering its rootmean-square (RMS). In that case, a division by $\sqrt{2}$ is needed for tonal vibrations at specific frequencies. Such scaling is merely scalar, hence inessential within the scope of this work. As a result, the use of the signal amplitude is preferred over its RMS in the remainder of this work.

Finally, the comfort vibration index (CVI) at a given frequency $\omega$ is defined as:

$$
\operatorname{CVI}(\omega)=W_{k}(\omega) A_{z \text {,seat }}(\omega)
$$

The comfort vibration index can give insight into the comfort evaluation as a result of the whole-body vibration experienced by a human, but it only tells one part of the whole story when the pilot is concerned. The next three sections describe the necessary additions to the conventional formula to achieve a generalized vibration measure of helicopter pilots. 


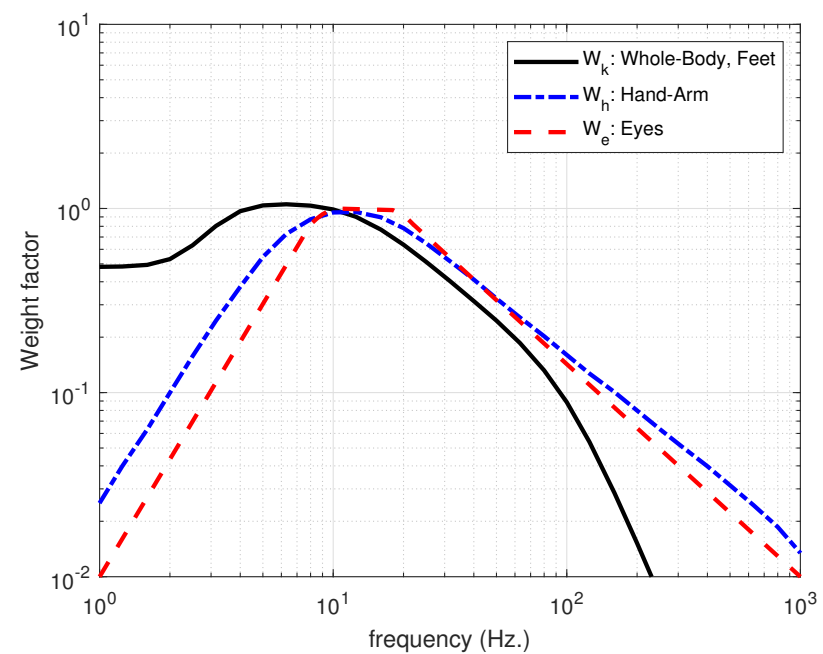

Figure 2. Frequency weights of vertical whole-body and feet vibration $\left(W_{k}\right)$, hand-arm vibration $\left(W_{h}\right)$, and eye vibration $\left(W_{e}\right)$.

\subsection{Handling Vibration Index}

The comfort vibration index considered in the previous section considers the motion of the whole body, ignoring that of specific body parts. However, arms and hands are connected to the trunk via joints and therefore have freedom to move, and the trunk itself is not a rigid body; in this context, it can be rather seen as a complex system of smaller bodies - the vertebrae-connected in series by complex compliant joints to compose the spine, with other sets of compliant bodies-the viscerae-in parallel to the spine itself. Hands themselves are prone to vibration-related risks [15]. Additionally, vibrations might even jeopardize flight safety if the motion transmitted to the hand is fed back to the control inceptors with suitable amplitude and delay, resulting in so-called unintended, adverse Rotorcraft-Pilot Couplings (RPC) [4]. Therefore, it is worth considering the vibration of the hand when evaluating the effects of vibration on helicopter pilots.

In a typical helicopter cockpit layout, the pilot controls the vehicle through collective and cyclic control inceptors, usually referred to as "sticks". Both hands of the pilot hold the grips, which are connected to the airframe structure through hinges and supports. As a result, both hands receive excitation from the cabin floor through two paths: i) from seat to trunk and then to the arms and hands, ii) directly from the control sticks. It can be reasonably assumed that high frequency vibrations are substantially filtered by the pilot's trunk and arm's admittance, whereas no such filtering occurs through the sticks.

There is no clear dominant direction for hands vibration; therefore, the magnitude of its translational acceleration is considered.

$$
A_{\text {hand }}(\omega)=\sqrt{A_{x, \text { hand }}^{2}(\omega)+A_{y, \text { hand }}^{2}(\omega)+A_{z, \text { hand }}^{2}(\omega)}=\left\|\mathbf{A}_{\text {hand }}\right\|
$$

Frequency weighting for human sensitivity to hand-arm vibration is available in the ISO-8041 standard [16]. It is presented in Figure 2 as $W_{h}$. The pilot controls two sticks, one for the cyclic and the other for the collective; as such, they should be both included in the formulation. Then, a handling vibration index (HVI) can be formulated, similar to the comfort vibration index of Equation (6), namely:

$$
\operatorname{HVI}(\omega)=W_{h}(\omega)\left(c_{\text {col }} A_{\text {hand,col }}(\omega)+c_{\text {cyc }} A_{\text {hand,cyc }}(\omega)\right)
$$

where $A_{\text {hand,col }}$ and $A_{\text {hand,cyc }}$ are the accelerations of the hands on the collective and cyclic grips, respectively, weighted by the related coefficients $c_{\mathrm{col}}$ and $c_{\mathrm{cyc}}$, with $c_{\mathrm{col}}+c_{\mathrm{cyc}}=1$. 
Without excessive loss of generality, in this work an average of both contributions is considered, with $c_{\mathrm{col}}=c_{\mathrm{cyc}}=1 / 2$. The resulting HVI is thus defined as:

$$
\operatorname{HVI}(\omega)=W_{h}(\omega) \frac{A_{\text {hand,col }}(\omega)+A_{\text {hand,cyc }}(\omega)}{2}=W_{h}(\omega) A_{\text {hand }}(\omega)
$$

\subsection{Visual Vibration Index}

Considering a typical cockpit, the whole body and instrument panel vibrate as a consequence of airframe vibrations. The human body vibrations are transmitted to the skull through the spine, and from the skull to the eyes, causing the motion of the retinal image [17]. The displays are excited through an independent load-path. As a result, relative motion occurs between the eye and the visual displays, which is the combination of two independent motions. Increasing levels of vibration degrade the display reading performance of the pilot, causing reading errors and longer response times [5]. Either the eye vibration or that of the display screens can dominate; however, it is likely that both contribute [18]. The degradation is proportional to the amplitude of the vibration [19], and to its frequency, as detailed in the following. Since this work focuses on human biodynamic aspects of vibration assessment, the panel vibration is not considered.

In the case of involuntary motion of head and eyes, the identification of the dynamic response from measurements becomes more difficult. For this reason, eye response characteristics in literature present significant variability in the critical frequencies greater than comfort-focused measurements on seat surface. Nevertheless, similar trends appear, which has been confirmed by independent studies. For example, results presented by Ohlbaum [20] reported that the eye response, relative to the skull, starts increasing at about $12 \mathrm{~Hz}$ and shows a peak in the vicinity of $18 \mathrm{~Hz}$. Otherwise, it follows the skull without any substantial amplification or attenuation. Similarly, Ishitake [21] presented a maximum in degradation of visual acuity at a frequency of $12.5 \mathrm{~Hz}$. In another work, significant contribution of human biodynamics on visual performance degradation above $10 \mathrm{~Hz}$ was found by Collins [22]. A frequency of $12 \mathrm{~Hz}$ is also reported as the target for the effect of vibration on visual acuity in a combined positive $G$ maneuver and sustained vibration [23]. Lewis and Griffin [24] extended the range for appreciable effects of vibration on visual performance up to $31.5 \mathrm{~Hz}$.

According to the literature surveyed in the previous paragraph, appreciable sensitivity to frequency is reported for the eyes between 12 and $18 \mathrm{~Hz}$, remaining appreciable up to $30 \mathrm{~Hz}$. Although there is no standardized frequency weighing for eye vibrations, these findings can be used to determine a sensitivity curve for the vibration of the eyes. Using the shapes defined for whole-body and hand-arm frequency weights of Figure 2, a frequency weighting for the eye is formed. It is presented in Figure 2, along with the others. The magnitude of the eye vibration is multiplied by this frequency weight $\left(W_{e}\right)$ and a visual vibration index (VVI) is readily obtained:

$$
\operatorname{VVI}(\omega)=W_{e}(\omega) A_{\text {eye }}(\omega)
$$

where $A_{\text {eye }}(\omega)$ is the norm of the vertical acceleration of the eye.

\subsection{Foot Vibration Index}

The other limbs that may be adversely affected by cockpit vibrations are the feet. In a typical helicopter cockpit, the feet act on the pedals that control the tail rotor collective pitch and remain in contact with them at all times. Any acceleration on the pedals is transmitted to the feet and interacts with the biomechanical impedance of the lower body. On the other side, the thighs are connected to the upper body, which is another load path. Therefore, the lower body, composed of legs and feet is an important part of the vibrations received by the pilot. The frequency weight is the same used for whole-body vibration, which is presented as $W_{k}$ in Figure 2. Then, similar to the above indices, a foot vibration one can be defined as 
the product of foot acceleration by the corresponding frequency weight function:

$$
\operatorname{FVI}(\omega)=W_{k}(\omega) A_{\text {foot }}(\omega)
$$

\subsection{Generalized Vibration Index}

A generalized vibration index (GVI) is proposed, which is composed of the indices described in the previous sections: (i) comfort vibration index (CVI), (ii) handling vibration index (HVI), (iii) visual vibration index (VVI), and (iv) foot vibration index (FVI). They are linearly combined with a further set of weight coefficients:

$$
\mathrm{GVI}=\mathrm{c}_{\mathrm{C}} \mathrm{CVI}+\mathrm{c}_{\mathrm{H}} \mathrm{HVI}+\mathrm{c}_{\mathrm{V} V \mathrm{VI}}+\mathrm{c}_{\mathrm{F}} \mathrm{FVI}
$$

where $c_{C}, c_{H}, c_{V}, c_{F} \geq 0$ are referred to as contribution weights, which determine the relative importance of comfort, handling, visual, and foot vibration indices in the general index. To normalize the GVI, the sum of these weights are set to unity:

$$
\mathrm{c}_{\mathrm{C}}+\mathrm{c}_{\mathrm{H}}+\mathrm{c}_{\mathrm{V}}+\mathrm{c}_{\mathrm{F}}=1
$$

As a result, the proposed GVI includes:

- acceleration estimates of the four contributions; namely the whole-body, the hand-arm (average of both), the eyes (symmetric motion), and the feet (symmetric motion);

- the frequency weights of these contributions that reflect human perception, which are determined by the standards or from the literature;

- a set of weight coefficients, which allow for the alteration of the relative importance of the specific indices in the formulation, when needed.

\section{Analysis Model}

This section explains how a detailed simulation model is built to demonstrate the evaluation of helicopter pilot vibration exposure using the suggested generalized vibration index. In principle, the construction of the GVI does not depend on how the required accelerations are gathered. It could be evaluated, for example, using experimentally measured accelerations. In the following, accelerations are rather computed using a realistic modeling framework for rotorcraft aeromechanics and pilot biodynamics, for the lack of actual measurements on the one hand, but also, on the other one, to exemplify how its numerical evaluation with sufficiently detailed, yet still relatively simple models, could be proficiently used in support of helicopter design, taking the effects of the inevitable vibrations in due account.

\subsection{Helicopter Model}

All contributors to the GVI require the calculation of the accelerations from the vibration source to seat, hands, eyes, and feet. For a rotorcraft, this can be achieved using a comprehensive model that includes:

- a high fidelity aeroelastic helicopter model for realistic vibratory loads estimation and a complete transmission to the pilot seat;

- models of human biodynamics to involve amplification and attenuation of vibrations as they propagate through the body;

- a versatile coupling of these two models, namely human biodynamics and helicopter aeroelasticity.

MASST (Modern Aeroservoelastic State Space Tools) is a tool developed at Politecnico di Milano for the analysis of compact, yet complete modular models of linearized aeroservoelastic systems $[25,26]$. MASST collects rotorcraft subcomponents developed in any source of choice, however complex, and assembles them using the Craig-Bampton Component Mode Synthesis (CMS) method [27]. The assembled mode is then cast in state-space form. This approach is very practical to formulate helicopter subcomponents 
(rotors, airframe, etc.) in their most suitable environments (e.g., Comprehensive Rotorcraft Analysis solvers for rotor aeromechanics, Finite Element solvers for airframe structural dynamics, etc.) and combine them in a rather versatile mathematical environment to form a complete model of arbitrary complexity.

After assembly, the model is cast into a quadruple of matrices A, B, C, D that define the system in state-space form:

$$
\begin{array}{r}
\dot{\mathbf{x}}=\mathbf{A x}+\mathbf{B f} \\
\mathbf{y}=\mathbf{C x}+\mathbf{D f}
\end{array}
$$

where vector $\mathbf{x}$ contains the states of the system, vector $\mathbf{y}$ contains the system output (accelerations of selected points, in the case at hand), and vector $\mathbf{f}$ collects the inputs (vibratory loads induced by periodic aerodynamic boundary conditions).

Since rotorcraft models may show a significant dependence on the operational conditions in which they are linearized, MASST can efficiently interpolate the overall state-space matrices in a generic configuration from the corresponding linear models evaluated in the space of prescribed parameters (e.g., trim conditions).

In the Laplace domain, the resulting model produces the input-output relationship:

$$
\mathbf{y}(s)=\left[\mathbf{C}(s \mathbf{I}-\mathbf{A})^{-1} \mathbf{B}+\mathbf{D}\right] \mathbf{f}(s)=\mathbf{G}(s) \mathbf{f}(s) .
$$

where $\mathbf{I}$ is the identity matrix and $\mathbf{G}(s)$ is the resulting transfer matrix. Therefore, the effective evaluation of a generalized vibration measure for helicopter pilots is supported by MASST in terms of:

1. flexibility in the source of subcomponent formulation;

2. mid- to high-fidelity overall modeling through subcomponent assembly;

3. capability of defining sensor-force relationships between arbitrary structural points;

4. ability to export proper models suitable for the efficient evaluation of accelerations at selected positions resulting from the defined inputs.

In this section, an accurate helicopter model is built based on data representative of a generic, medium weight helicopter with an articulated five blade main rotor. A snapshot of the physical kinematic variables of the virtual helicopter model is shown in Figure 3.

The state-space model includes:

- $\quad$ six rigid body degrees of freedom, associated with the flight mechanics of the helicopter;

- $\quad$ flight mechanics aerodynamic stability derivatives of the airframe, estimated using CAMRAD/JA (CAMRAD/JA is a popular commercial rotorcraft comprehensive analysis software, produced by Johnson Aeronautics);

- normal vibration modes of the airframe covering the frequency range of interest, which are extracted from MSC/NASTRAN (MSC/NASTRAN is a commercial Finite Element solver);

- $1.5 \%$ proportional structural damping superimposed in MASST on the airframe vibration modes;

- the first two bending modes and the first torsion mode of the main and tail rotors, including the aerodynamic matrices in multiblade coordinates obtained, averaged to eliminate azimuth dependence when trimmed in forward flight, obtained using CAMRAD/JA;

- the transfer functions of main and tail rotor servo actuators directly formulated in Matlab/Simulink, considering servo-valve dynamics and dynamic compliance [28];

- $\quad$ the input, B, and output, $\mathbf{C}$ and $\mathbf{D}$ matrices corresponding to the locations of the nodes for the sensors and the applied loads, directly defined in MASST.

- detailed biodynamic models of the pilot, coupled to the helicopter model at the cockpit location. 
In the presented analysis, the input is a harmonic vertical force acting at the main hub, spanning the desired frequency range. Sensors are defined defined at the pilot's seat and foot locations.

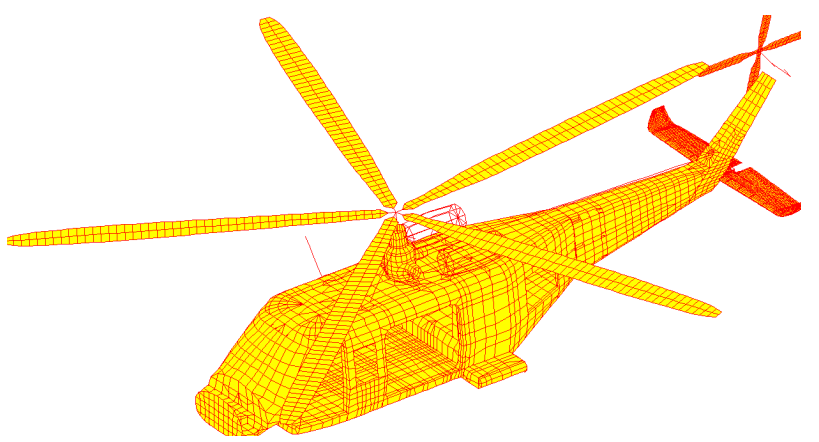

Figure 3. Snapshot of the helicopter model.

\subsection{Biodynamic Model}

The proposed GVI requires the evaluation of the acceleration levels of the seat surface, of the pilot's eyes, of the collective control stick, and of the pilot's feet. The human body response to the input acceleration brings therefore a significant contribution to the GVI. The problem can be tackled through the biomechanical modeling of the pilot's body and, as it is always the case with numerical modeling, the choice of the level of broadness and depth in the analysis has a profound impact on the efficiency and completeness of the analysis.

The choice must balance on one side the ability of the biodynamic models to correctly represent the variability of the response of different subjects, having different anthropometric characteristics (primarily age, height, weight) and, in general, different poses when seated in the cockpit holding the control inceptors; on the other side, the resulting model complexity, affecting the computational time, the time needed to adjust the model to a different subject and operating conditions, and also the effort needed to maintain and continuously update the model itself.

In the literature, a wide spectrum of different approaches can be found: from simpler, lumped-parameter models only conceptually representing the related body parts and having a few to several degrees of freedom to fully nonlinear, physics-based finite element models with millions of degrees of freedom [29]. Toward the middle of the complexity spectrum multibody models are found: they offer an exceptional ability to consider the effects of nonlinearities, especially those arising from 3D geometry, combined with a strong physics-based approach and thus the ability to be scaled easily to represent different subjects, with a fully parametrized approach [30]. The latter approach was selected as the most appropriate for the pilot upper body [31], since it is the section most affected by parameter variability and geometric nonlinearities arising from 3D pose variations.

A mixed approach has been deemed the most appropriate in this context. A complete model of the pilot body has been obtained connecting the following four submodels of different complexity:

1. a complete multibody model of the upper limbs;

2. a reduced order model of the spine and torso, obtained through modal condensation techniques from a complete multibody model;

3. a lumped parameter model of the lower limbs;

4. a simplified representation of the dynamics of the pilot eyes' vertical motion, obtained from experimentally identified transfer functions.

The multibody models have been developed using the free multibody software MBDyn (http: / / www.mbdyn.org / last accessed January 2021) [32], also developed by the authors. They are connected directly in MBDyn, first extracting a reduced, Component Mode Synthesis (CMS) model of the spine using the boundary masses approach proposed 
in [33] and then connecting to the resulting modal element the full model of the left upper limb. The model is then augmented introducing the rigid-body representation of the collective inceptor, joined to the left hand. More details about the employed procedure can be found in the following sections.

The resulting multibody model of the upper part of the body, shown in Figure 4, is then used to extract, via numerical experiments, the input-output transfer function relating the seat acceleration input $\ddot{z}_{s}(s)$ to the collective acceleration $\ddot{z}_{c}(s)$ and the head acceleration $\ddot{z}_{h}(s)$. The resulting reduced, state-space, submodel is then joined to the other ones in MASST.

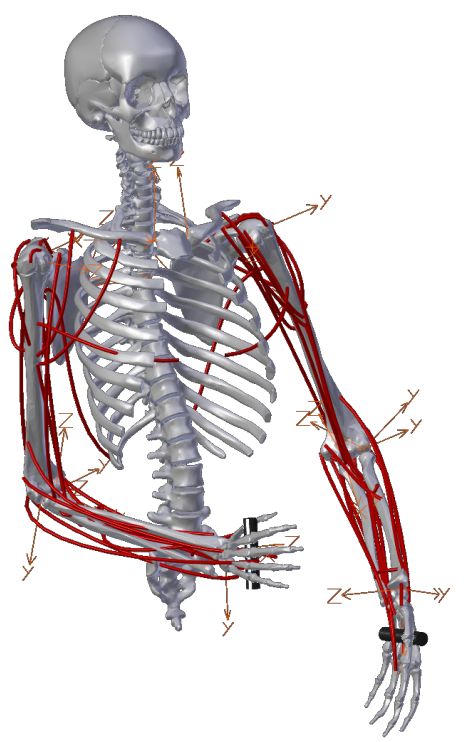

Figure 4. CAD representation of the human upper body multibody model, with muscle actuators highlighted and upper limbs nodes indicated by triads. Vertebral nodes are not highlighted to avoid cluttering.

\subsubsection{Multibody Model of Spine and Torso}

The model has been under development by the authors' research group for several years; only a brief description is proposed in this section, whereas the interested reader is referred to the available literature for more details [30,31,34]. The basic structure of the model is derived from previous efforts, in particular from the model proposed by Kitazaki and Griffin [35]: it includes 34 rigid bodies introduced in correspondence to the each vertebra, representing the associated trunk sections, from C1 to S1. Each vertebral node is connected to the neighboring ones by ideal kinematic constraints removing the anteroposterior and medio-lateral relative degrees of freedom. They are further interconnected by linear viscoelastic bushing elements, representing the compliance and damping properties arising from both the action of the muscles in posture control and the contribution of the tissues interposed between vertebrae themselves.

Seven additional rigid bodies take into account the motion of the visceral masses; they are connected via viscoelastic $1 \mathrm{D}$ elements, acting in the antero-posterior direction, to the vertebral nodes between T10 and S1, and among them by a further set of viscoelastic 1D elements, acting along the supero-inferior direction.

The head is supposed rigid; it is thus introduced as a single rigid body connected to $\mathrm{C} 1$ with the same algebraic joints used for the inter-vertebral connections. The pelvis is also introduced as a single rigid body connected to S1. In this case, the connection is rigid; therefore, the mass of the pelvis is simply added to the mass of the S1 section. The compliance of the buttock tissue is taken into account by adding a node rigidly connected to the seat cushion, constrained to the S1 node in order to allow only the supero-inferior translation and the rotations in the sagittal and coronal plane; a 3D viscoelastic element reacts the allowed relative motion. 
Subject-specific models can be generated starting from their basic anthropometric parameters: age, weight, height or Body Mass Index (BMI), and sex. The model is fully parametric and a dedicated scaling procedure has been developed to adjust the geometric, inertial, and structural parameters of the different subjects $[30,36]$.

At this point, the subject-specific spine model can be reduced using the boundary masses approach proposed in [33]: large fictitious masses are added in correspondence of the interface with the model of the upper limbs, i.e., in the shoulder girdles. Mode shapes are then computed directly from the multibody model [37,38], and the effect of the added masses is removed from the resulting generalized mass and stiffness matrix. In the reduced model considered in this work, only the mode shapes lying entirely in the sagittal plane have been retained.

\subsubsection{Upper Limbs Model}

The upper limbs model is a multibody representation of the hands, forearms, and arms. Developed following the work of Pennestrì et al. [39], it was initially presented in [40], and subsequently underwent significant evolution to its present form [30].

In this work, it is composed by four nodes for each limb, associated with the bony structures of the humerus, ulna, radius, and hand. The latter is considered as a single rigid body, as during piloting it is usually involved in gripping tasks. The nodes are connected by ideal algebraic constraints. A spherical joint models the shoulder complex, connecting in this case the humerus with the torso; a revolute joint located at the humerus distal epicondyle connects the ulna and the humerus; a spherical joint, also located at the humerus distal epicondyle, connects the humerus and the radius; a point-on-line constraint connects the ulna and the radius, near the radius distal epicondyle; finally, a Cardano joint located at the radius distal epicondyle connects it to the hand. The location of the point-on-line constraint between the radius and the ulna is such as to leave the mechanical axes of the two bony structures parallel when the forearm is in the neutral anatomical configuration.

The bony structures are actuated by $251 \mathrm{D}$ viscoelastic muscle actuators, following the simplified Hill-type model presented in [39]. The model is both kinematically underdetermined and overactuated: dedicated solution strategies have been devised to solve the kinematic underdeterminacy directly at the position level and to estimate both the baseline muscles' activation, i.e., the minimum required one to maintain the reference pose, and the reflexive activation, introduced by the Central Nervous System (CNS) to perform active posture control and impedance modulation.

\subsubsection{Ocular Dynamics}

The biodynamics model illustrated in the previous section includes the motion of the head as a single rigid body connected to an elastic model of the spine. This model is reasonable to capture the response of the head or that of an eye that rigidly follows the head. However, additional local motion between the head and the eye can occur, which is referred to as the "compliant eye" motion. This relative motion of the eye with respect to the skull is expected to cause an involuntary motion of the eye, therefore resulting in a blurring perception of the visual cues. The compliant eye dynamics with respect to the head can be modeled using the same computational techniques that are used in biodynamic modeling (see for example Ref. [41]). However, cognitive processes intervene with the dynamical behaviour of the eye, and a fully mechanical model can be misleading. A complete model of ocular dynamics involving the cognitive interaction requires a dedicated biomechanical research and thus is beyond the scope of the present work. Therefore, empirical transfer functions of the eye, which are obtained via experimental measurements, have been selected to account for ocular dynamics. This is much more realistic than completely ignoring its presence, in the absence of a complete and detailed model of the eye.

Among the available studies addressing the ocular dynamics, Ref. [42] is preferred, since it reports the head to eye frequency response, which includes magnitude, phase, and 
their standard deviation. As a result, the relative transfer function from the head to the eye can be multiplied by the head acceleration resulting from the spine-trunk model to obtain the absolute acceleration of the eye. The resulting relative eye motion of a compliant eye model is compared to that of a rigid eye one in Figure 5.

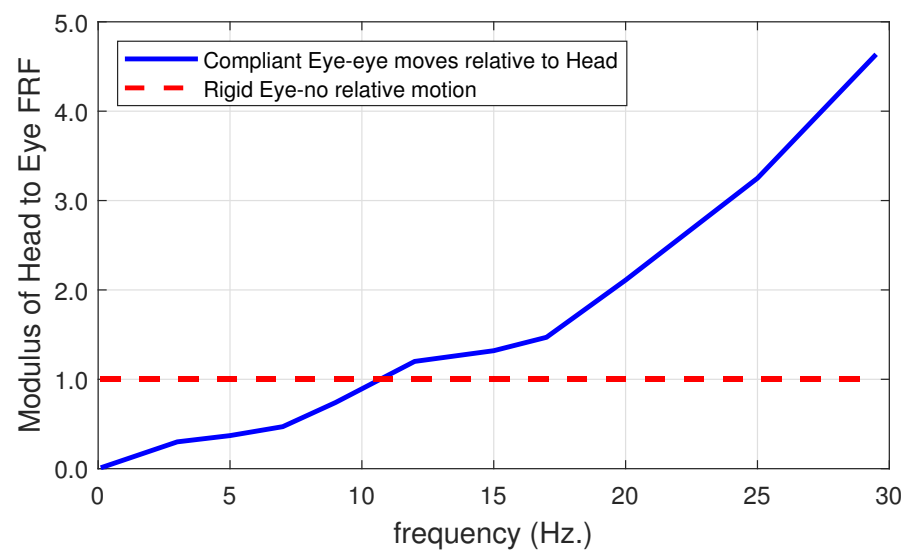

Figure 5. Modulus of the head to eye frequency response function.

\subsubsection{Lower Limb Model}

Considering their size, pilot feet can be considered as rigid bodies that are exposed to vibrations coming from the cockpit pedals. However, this would be a simplified picture since each foot is connected to the main body via calf and thigh and hence interacts with the pilot, seat, and floor. Still, the legs and feet, namely lower limb, are less complex as compared to the hands and arms for a helicopter pilot since they are supported by the seat and pedals. Although such considerations on biodynamic modeling techniques are valid and ought to be used in this framework, a lumped model from a helicopter-related study is selected instead from Ref. [43]. The lumped model of lower limb is presented in Figure 6, whose data is reported in Table 2.

The connection between the feet and the pedals requires attention. It can have two states: (i) the feet rest on the pedals as the trunk rests on seat, and (ii) they move freely in differential motion. While the former happens in most of the flight time, the latter happens occasionally in short periods of time to act on the tail rotor command. Therefore, the resting state is assumed to be the nominal state in this work. This suggests that the feet can be considered to be rigidly connected to the floor via the bottom part of the pedals.

Table 2. Numerical values for the human lower limb model of Figure 6, adapted from Ref. [43].

\begin{tabular}{lccc}
\hline & $\boldsymbol{m}_{\boldsymbol{i}} \mathbf{( k g )}$ & $\boldsymbol{c}_{\boldsymbol{i}} \mathbf{( \mathbf { k N ~ s ~ m }} \mathbf{- 1}^{\mathbf{1}} \mathbf{)}$ & $\boldsymbol{k}_{\boldsymbol{i}}\left(\mathbf{k N ~ m}^{\mathbf{- 1}} \mathbf{)}\right.$ \\
\hline Thigh (subscript $t)$ & 9.8 & 44.3 & 53.8 \\
Calf (subscript $c)$ & 3.8 & 43.7 & 54.4 \\
Foot (subscript $f$ ) & 1.0 & 63.1 & 51.4 \\
\hline
\end{tabular}




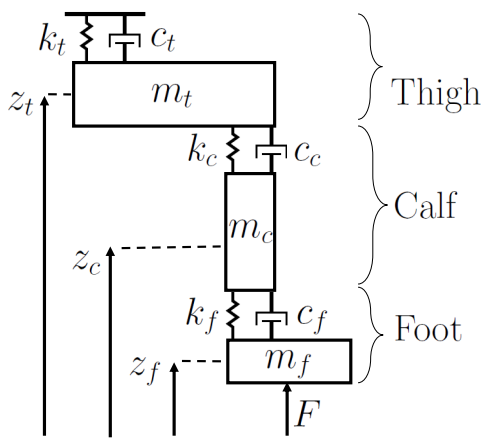

Figure 6. Human lower limb lumped parameter model (adapted from Ref. [43]; data in Table 2).

\subsection{Pilot Seat}

In a helicopter cockpit, a seat-cushion system supports the pilot's body in their sitting posture. A properly designed interface can significantly reduce the vibrations received by the pilot and hence improve comfort. Since considering the pilot as directly connected to the bare floor is not deemed realistic, a seat and cushion is adapted from a helicopter application from the literature [44]. The model is composed of the seat structure and cushion, both of which are described as masses suspended by a spring and damper. The lumped model is sketched in Figure 7; data are reported in Table 3.

Table 3. Numerical values for the seat-cushion model of Figure 7.

\begin{tabular}{lccc}
\hline & $\boldsymbol{m}_{\boldsymbol{i}} \mathbf{( k g )}$ & $\boldsymbol{c}_{\boldsymbol{i}} \mathbf{( \mathbf { N ~ s ~ m } \mathbf { ~ } ^ { - \mathbf { 1 } } )}$ & $\boldsymbol{k}_{\boldsymbol{i}}\left(\mathbf{k N ~ \mathbf { ~ m } ^ { \mathbf { - 1 } } )}\right.$ \\
\hline Seat (subscript s) & $13.5^{1}$ & $750.00^{1}$ & $22.6^{1}$ \\
Cushion (subscript $c)$ & $1.0^{2}$ & $159.00^{1}$ & $37.7^{1}$ \\
\hline
\end{tabular}

${ }^{1}$ From Ref. [44]; ${ }^{2}$ assumed.

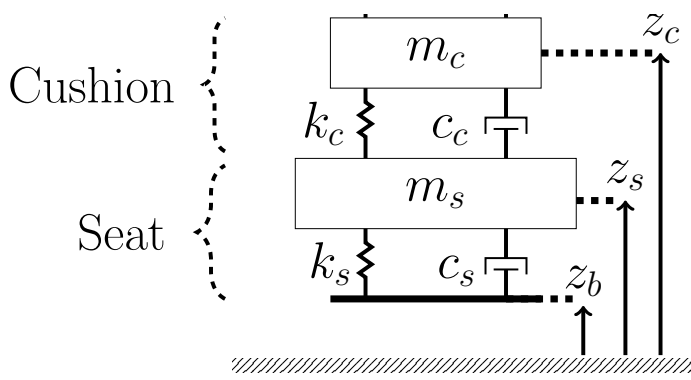

Figure 7. Cushion and seat model, interfacing the pilot's body to the cabin floor (adapted from [44]; data in Table 3).

\section{Results and Discussion}

In this section, the detailed and fully coupled helicopter-pilot model described in the previous sections is used to illustrate the evaluation of the generalized index for vibration exposure of helicopter pilots. The analyses are performed up to $30 \mathrm{~Hz}$, since above that frequency the amplitude of helicopter vibratory loads and human vibration sensitivity typically reduce. Therefore, vibrations above $30 \mathrm{~Hz}$ are not expected to cause significant consequences.

First, the generalized vibration index (GVI) is compared with the conventional one in Figure 8. The conventional index consists of a special case of the GVI, obtained by setting $\mathrm{c}_{\mathrm{C}}=1$ (and thus all the other coefficients equal to zero) in Equation (12). In the Figure, the GVI is evaluated using equal weight for comfort, handling, visual, and foot contributions $\left(c_{C}=c_{H}=c_{V}=c_{F}=0.25\right)$. The differences in magnitude are significant over the full range, except in the $10-20 \mathrm{~Hz}$ range, although the two curves share the location of the peaks. This may seem to be an obvious result, considering that the curves result 
from the dynamics of the same system, i.e., their transfer functions share the same poles. However, the curve associated with the GVI also includes the dynamics of the pilot's upper and lower body, the former in the hand-arm and eye contribution, and the latter in that of the feet-legs. This suggests that the eye, hand-arm, and foot-leg systems do not introduce additional resonances within the frequency range of interest. Instead, they amplify or dampen the vibrations transferred from the whole-body dynamics to the hand, eye, and feet, depending on the frequency.

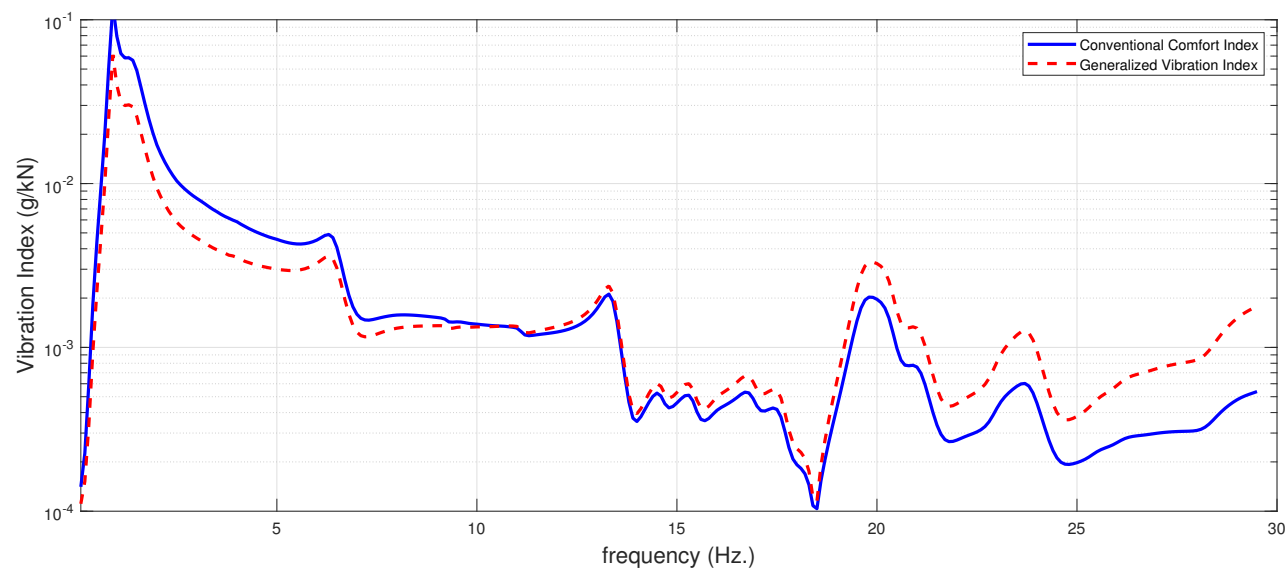

Figure 8. Proposed generalized vibration index, compared with conventional vibration exposure index for equal weights on comfort, handling, visual, and feet vibration indices.

Figure 9 shows the breakdown of the GVI of Figure 8 in its contributions, namely comfort, handling, visual, and foot vibration indices. In Figure 9, each contribution is shaded separately. One can observe that the CVI and FVI dominate up to $5 \mathrm{~Hz}$. Then, all four subindices give more or less equal contribution up to $20 \mathrm{~Hz}$. At higher frequencies, the visual index increases its contribution and dominate together with FVI. The major outcome of this breakdown is the frequency-wise explanation of the differences between the conventional index and the generalized index that was appreciated in Figure 8. A practical use could be to choose the vibration reduction solutions more precisely, targeting them to the most affected contribution. For example, over the frequency range where the CVI, i.e., whole-body vibration, dominates, a global isolation of the airframe or of the human body would be more appropriate. On the other hand, as happened in this particular case beyond $20 \mathrm{~Hz}$, the amplified VVI can be solved using display modulation (see for example [45]), which does not result in any weight penalty.

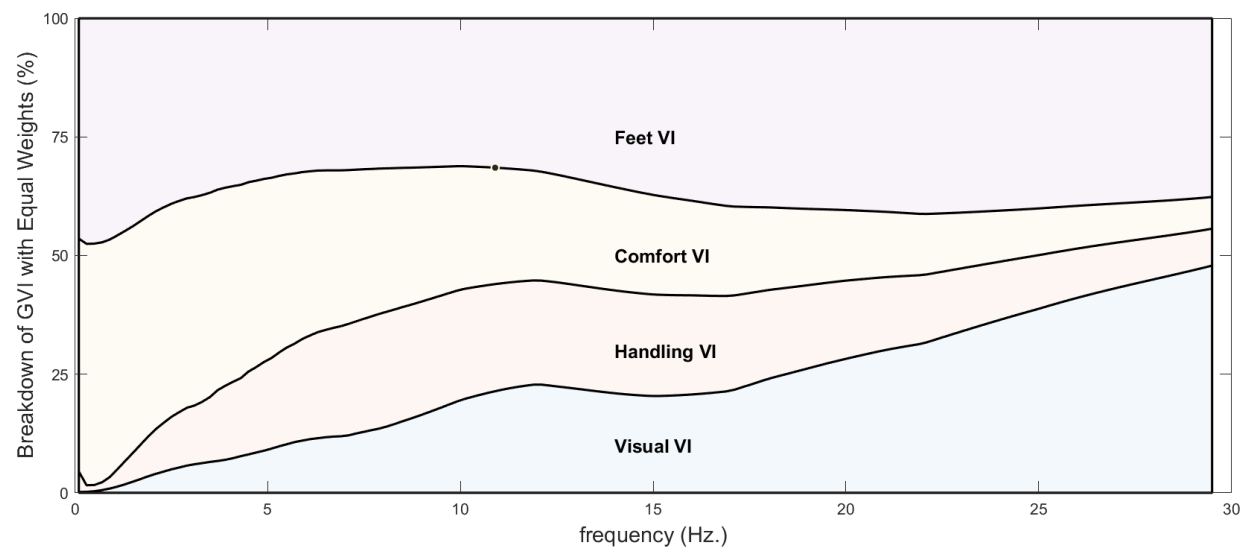

Figure 9. Contribution breakdown of generalized vibration index for equal weights on comfort, handling, visual, and feet vibration indices. 
The final analysis demonstrates how the GVI can be used to achieve a conservative vibration exposure assessment. In the nominal GVI formulation, equal contribution weights are used. However, each contribution can be given different priority, possibly depending on each individual's perception or other considerations related to the type of application, operation, or mission. In some cases, it may not be immediately clear whether equal weights should be given or not to all contributions. Therefore, the sensitivity of the nominal GVI formula to the contribution weights could help providing a more conservative-a more robust-assessment. For this purpose, the contribution weights $c_{i}$ are changed, while ensuring that $\sum_{i} c_{i}=1$, to obtain alternative GVIs. The area between the maximum and minimum GVI is shaded in the left plot of Figure 10. The difference from the equal-weight case, which assumes a $c_{i}=1 / 4$ for each contribution to the GVI, is significant under 6 $\mathrm{Hz}$ and above $22 \mathrm{~Hz}$. The two plots on the right of Figure 10 illustrate the values of the contribution weights for the maximum and minimum GVI values. For the specific problem under analysis, for example, the maximum GVI is dominated by the CVI up to $8 \mathrm{~Hz}$. Then, a broad frequency range is mainly influenced by the FVI; however, in this range the GVI value is smaller than that reached at the peak around $2 \mathrm{~Hz}$ and relatively insensitive to changes in weights of the individual contributions. In the rest of the plot, beyond $25 \mathrm{~Hz}$, the VVI takes the lead. Therefore, it can be stated that the equal-weight formulation is sensitive to the CVI when the frequency is low and to the VVI when the frequency is high but otherwise relatively insensitive to a specific index.
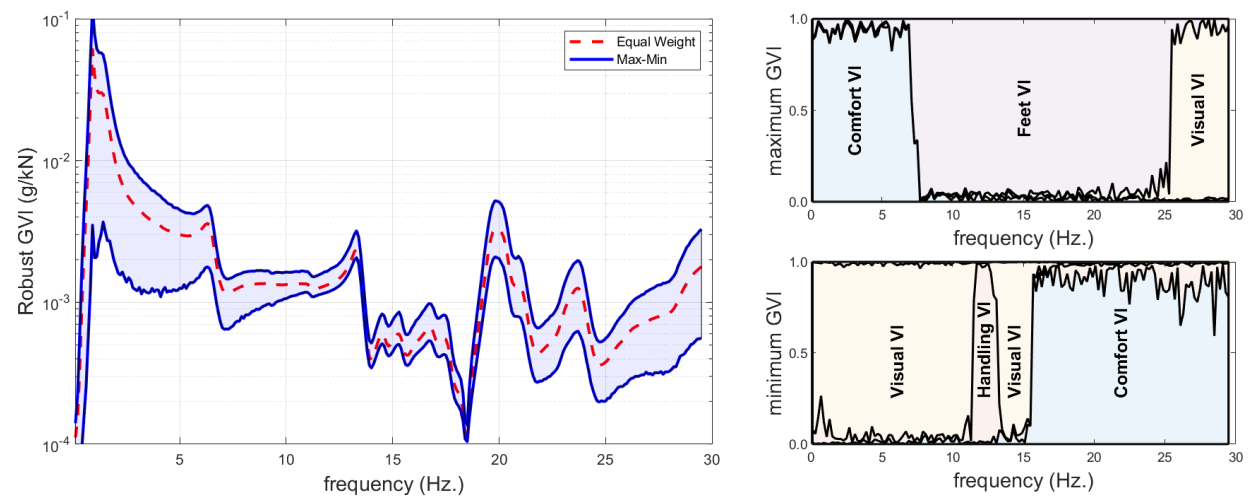

Figure 10. Robust generalized vibration index using different weights on comfort, handling, visual, and feet vibration indices.

It is worth noticing that the excitation sources can induce vibrations at different frequencies. As such, a broadband frequency sweep is useful to understand the whole picture of pilot vibration exposure. On the other hand, the main and persistent source of excitation in helicopters occurs at the frequency $\omega=N_{b} \Omega$, where $\Omega$ is the main rotor angular velocity and $N_{b}$ is the number of blades, also referred to as $N_{b} /$ rev vibrations. In evaluating the previously discussed curves one can focus on a narrow band of frequencies centered in $N_{b} \Omega$, to address the vibration exposure at $N_{b} /$ rev. In the model used for the present analysis, for example, $\Omega \approx 5 \mathrm{~Hz}$ and $N_{b}=5$; thus, $\omega \approx 25 \mathrm{~Hz}$. In the vicinity of this frequency, Figure 8 shows that the GVI exceeds by far the CVI. In fact, Figure 9 shows that the CVI only accounts for about $10 \%$ of the overall GVI. Additionally, Figure 10 shows that, with respect to the maximum GVI, we are at the verge of a change in relative importance between the FVI at $\omega<25 \mathrm{~Hz}$ and VVI at $\omega>25 \mathrm{~Hz}$. Although by no means such statements can be considered of general validity, they clearly illustrate the type of considerations that the proposed generalized index can support. 


\section{Conclusions}

This work investigated the benefits of transforming the classical vibration assessment measure into a generalized formulation. The suggested generalized vibration index (GVI) can be obtained in three steps:

- the acceleration contribution from the whole-body, hand-arm, eye, and foot or the GVI contributions, are estimated in a coupled helicopter-human framework;

- frequency weights are applied to the value of mechanical acceleration of each GVI contribution to reflect human sensitivity;

- the resulting frequency weighted accelerations are linearly summed by adding another coefficient that reflects the importance of each GVI contribution.

A modular aeroservoelastic simulation framework is used to obtain a coupled highfidelity helicopter-human model. The proposed GVI was demonstrated with its possible advantages over the classical one. It was shown that:

- the GVI can be significantly different than the conventional assessment. Therefore, the adverse effects of vibrations on the pilot can be comprehensively addressed;

- hand-arm, foot, and eye vibration are directly related to the flight safety and can significantly contribute to the GVI; therefore, the proposed GVI comprises safety in addition to the comfort and health of the pilot;

- a risk assessment of vibration on the contributed human body parts can be made by breaking down the GVI into its contributions. As a result, a precise evaluation of vibration reduction solutions can be performed, depending on the frequency of the vibration source and the most affected body part at that frequency;

- sensitivity of GVI formulation to the participation of each contribution can be evaluated using a robust version of the GVI.

- the modular framework, which helps to obtain the overall high-fidelity analysis model, supports the effectiveness of GVI formulation by providing the full structural load paths and realistic aeroelastic behavior of the vehicle.

Author Contributions: Conceptualization, A.T.; methodology, A.T., A.Z., A.C. and P.M; software, A.T, A.Z. and A.C; formal analysis, A.T.; investigation, A.T.; writing-original draft preparation, A.T. and A.Z.; writing-review and editing, A.T., A.Z. A.C. and P.M.; visualization, A.T.; supervision, P.M.; project administration, P.M.; funding acquisition, P.M. All authors have read and agreed to the published version of the manuscript.

Funding: This work received partial support by Leonardo Helicopter Division. The authors acknowledge LHD for providing part of the data used in the analysis.

Institutional Review Board Statement: Not applicable.

Informed Consent Statement: Not applicable.

Data Availability Statement: Not applicable.

Conflicts of Interest: The authors declare no conflict of interest.

\section{References}

1. Ljungberg, J.K.; Neely, G. Stress, subjective experience and cognitive performance during exposure to noise and vibration. J. Environ. Psychol. 2007, 27, 44-54. [CrossRef]

2. Harrer, K.L.; Yniguez, D.; Maria, M.M.; Ellenbecker, D.; Estrada, N.; Geiger, M. Whole Body Vibration Exposure for MH-60S Pilots. In Proceedings of the 43rd Annual SAFE Association Symposium, Salt Lake City, UT, USA, 24-26 October 2005.

3. Masarati, P.; Quaranta, G.; Gennaretti, M.; Serafini, J. An Investigation of Aeroelastic Rotorcraft-Pilot Interaction. In Proceedings of the 37th European Rotorcraft Forum, Vergiate and Gallarate, Italy, 13-15 September 2011.

4. Quaranta, G.; Tamer, A.; Muscarello, V.; Masarati, P.; Gennaretti, M.; Serafini, J.; Colella, M.M. Rotorcraft Aeroelastic Stability Using Robust Analysis. CEAS Aeronaut. J. 2014, 5, 29-39. [CrossRef]

5. Griffin, M.J. Eye Motion during Whole-Body Vertical Vibration. Hum. Factors 1976, 18, 601-606. [CrossRef] [PubMed]

6. ISO. ISO Mechanical Vibration and Shock-Evaluation of Human Exposure to Whole-Body Vibration; Technical Report ISO2631-1; ISO: Geneva, Switzerland, 1997. 
7. Leatherwood, J.D.; Clevenson, S.A. A Design Tool for Estimating Passenger Ride Discomfort within Complex Ride Environments. J. Hum. Factors Ergon. Soc. 1980, 22, 291-312. [CrossRef]

8. AMCOM. Requirements for Rotorcraft Vibration Specifications, Modeling and Testing; ADS-27A; US Army AMCOM: Redstone Arsenal, AZ, USA, 2006.

9. Chen, Y.; Yapa, U.S.; Price, A.; Wickramasinghe, V. Evaluation of Aircrew Whole-Body Vibration and Mitigation Solutions for Helicopter Flight Engineers. In Proceedings of the AHS 73rd Annual Forum, Fort Worth, TX, USA, 9-11 May 2017.

10. Law, A.H.; Beatty, H.E.W.; Keillor, J.; Wickramasinghe, V. Pilot Head and Neck Response to Helicopter Whole Body Vibration and Head-Supported Mass. In Proceedings of the AHS 73rd Annual Forum, Fort Worth, TX, USA, 9-11 May 2017.

11. Tamer, A.; Muscarello, V.; Masarati, P.; Quaranta, G. Evaluation of vibration reduction devices for helicopter ride quality improvement. Aerosp. Sci. Technol. 2019, 95, 105456. [CrossRef]

12. Tamer, A.; Zanoni, A.; Cocco, A.; Masarati, P. Generalized Measure of Vibration Exposure for Helicopter Pilots. In Proceedings of the 45th European Rotorcraft Forum, Warsaw, Poland, 17-20 September 2019.

13. Rath, T.; Fichter, W. A Closer Look at the Impact of Helicopter Vibrations on Ride Quality. In Proceedings of the AHS 73rd Annual Forum, Fort Worth, TX, USA, 9-11 May 2017.

14. Johnson, W. Rotorcraft Aeromechanics; Cambridge University Press: New York, NY, USA, 2013.

15. Mansfield, N. Human Response to Vibration; Taylor \& Francis: Abingdon, UK, 2004.

16. ISO. ISO Human Response to Vibration-Measuring Instrumentation; Technical Report ISO8041; ISO: Geneva, Switzerland, 2005.

17. Lewis, C.; Griffin, M. A review of the effects of vibration on visual acuity and continuous manual control, part II: Continuous manual control. J. Sound Vib. 1978, 56, 415-457. [CrossRef]

18. Nakashima, A.; Cheung, B. The Effects of Vibration Frequencies on Physical, Perceptual and Cognitive Performance; Technical Report DRDC Toronto TR 2006-218; DRDC: Toronto, ON, Canada, 2006.

19. O'Briant, C.R.; Ohlbaum, M.K. Visual Acuity Decrements Associated with Whole Body Plus or Minus Gz Vibration Stress. Aerosp. Med. 1970, 41, 79-82.

20. Ohlbaum, N.K. Mechanical Resonant Frequency of the Human Eye In Vivo. Ph.D. Thesis, Aerospace Medical Research Laboratory, Wright-Patterson Air Force Base, OH, USA, 1976.

21. Tatsuya Ishitake, Hideo Ando, Y.M.; Matoba, F. Changes of Visual Performance Indced by Exposure to Whole-body Vibration. Kurume Med. J. 1998, 45, 59-62. [CrossRef]

22. Collins, A.M. Decrements in Tracking and Visual Performance during Vibration. Hum. Factors 1973, 15, 379-393. [CrossRef]

23. Adelstein, B.D.; Beutter, B.R.; Kaiser, M.K.; McCann, R.S.; Stone, L.S.; Anderson, M.R.; Renema, F.; Paloski, W.H. Influence of Combined Whole-Body Vibration Plus G-Loading on Visual Performance; Technical Report NASA-TM-2009-215386; NASA: Washington, DC, USA, 2009.

24. Lewis, C.H.; Griffin, M.J. Predicting the effects of vibration frequency and axis, and seating conditions on the reading of numeric displays. Ergonomics 1980, 23, 485-499. [CrossRef]

25. Masarati, P.; Muscarello, V.; Quaranta, G. Linearized Aeroservoelastic Analysis of Rotary-Wing Aircraft. In Proceedings of the 36th European Rotorcraft Forum, Paris, France, 7-9 September 2010.

26. Masarati, P.; Muscarello, V.; Quaranta, G.; Locatelli, A.; Mangone, D.; Riviello, L.; Viganò, L. An Integrated Environment for Helicopter Aeroservoelastic Analysis: the Ground Resonance Case. In Proceedings of the 37th European Rotorcraft Forum, Vergiate and Gallarate, Italy, 13-15 September 2011.

27. Craig, Jr., R.R.; Bampton, M.C.C. Coupling of Substructures for Dynamic Analysis. AIAA J. 1968, 6, 1313-1319. [CrossRef]

28. Merritt, H.E. Hydraulic Control Systems; John Wiley \& Sons: New York, NY, USA, 1967.

29. Mohajer, N.; Abdi, H.; Nahavandi, S.; Nelson, K. Directional and sectional ride comfort estimation using an integrated human biomechanical-seat foam model. J. Sound Vib. 2017, 403, 38-58. [CrossRef]

30. Zanoni, A.; Cocco, A.; Masarati, P. Multibody dynamics analysis of the human upper body for rotorcraft-pilot interaction. Nonlinear Dyn. 2020, 102, 1517-1539. [CrossRef]

31. Tamer, A.; Zanoni, A.; Muscarello, V.; Cocco, A.; Quaranta, G.; Masarati, P. Biodynamic Modeling Techniques for Rotorcraft Comfort Evaluation. Aerotec. Missili Spaz. 2019, 98, 147-158. [CrossRef]

32. Masarati, P.; Morandini, M.; Mantegazza, P. An efficient formulation for general-purpose multibody/multiphysics analysis. J. Comput. Nonlinear Dyn. 2014, 9, 041001. [CrossRef]

33. Karpel, M.; Raveh, D. Fictitious Mass Element in Structural Dynamics. AIAA J. 1996, 34, 607-613. [CrossRef]

34. Masarati, P.; Quaranta, G.; Zanoni, A. A Detailed Biomechanical Pilot Model For Multi-Axis Involuntary Rotorcraft-Pilot Couplings. In Proceedings of the 41st European Rotorcraft Forum, Munich, Germany, 1-4 September 2015.

35. Kitazaki, S.; Griffin, M.J. A modal analysis of whole-body vertical vibration, using a finite element model of the human body. $J$. Sound Vib. 1997, 200, 83-103. [CrossRef]

36. Zanoni, A.; Masarati, P. Geometry generation and benchmarking of a complete multibody model of the upper limb. In Proceedings of the Fourth Joint International Conference on Multibody System Dynamics - IMSD 2016, Montréal, QC, Canada, 29 May-2 June 2016.

37. Masarati, P. Direct Eigenanalysis of Constrained System Dynamics. Proc. IMechE Part K J. Multi-Body Dyn. 2009, $223,335-342$. [CrossRef] 
38. Ripepi, M.; Masarati, P. Reduced Order Models Using Generalized Eigenanalysis. Proc. IMech. E Part K J. Multi-Body Dyn. 2011, 225, 1-14. [CrossRef]

39. Pennestrì, E.; Stefanelli, R.; Valentini, P.P.; Vita, L. Virtual musculo-skeletal model for the biomechanical analysis of the upper limb. J. Biomech. 2007, 40, 1350-1361. [CrossRef]

40. Masarati, P.; Quaranta, G.; Zanoni, A. Dependence of helicopter pilots' biodynamic feedthrough on upper limbs' muscular activation patterns. Proc. IMechE Part K J. Multi-Body Dyn. 2013, 227, 344-362. [CrossRef]

41. Pascolo, P.; Carniel, R.; Grimaz, S. Dynamical models of the human eye and strabismus. Chaos Solitons Fractals 2009, 41, 2463-2470. [CrossRef]

42. Lee, R.A.; King, A.I. Visual vibration response. J. Appl. Physiol. 1971, 30, 281-286. [CrossRef] [PubMed]

43. Khaksar, Z.; Anavatti, S.; Shankar, K. The Vibration Simulation at the Helicopter Pilot's Body Segments in Vertical Direction. In Advances in Intelligent Systems and Computing; Springer: Cham, Switzerland, 2017. [CrossRef]

44. Choi, Y.T.; Wereley, N. Biodynamic Response Mitigation to Shock Loads Using Magnetorheological Helicopter Crew Seat Suspensions. J. Aircr. 2005, 42, 1288-1295. [CrossRef]

45. Kaiser, M.K.; Adelstein, B.D.; Anderson, M.R.; Beutter, B.R.; Ahumada, A.J., Jr; McCann, R.S. Stroboscopic Image Modulation to Reduce the Visual Blur of An Object being Viewed by An Observer Experiencing Vibration. U.S. Patent No 8,711,462, 29 April 2014 